\title{
Concise Commentary: Pocketing the Difference-Genetics and the Changing Paradigms of Diverticulitis Management
}

\author{
Des C. Winter ${ }^{1}$
}

Published online: 26 July 2019

(c) Springer Science+Business Media, LLC, part of Springer Nature 2019

The primacy of surgery for diverticulitis is an unassailable tenet of modern management that is unlikely to change in the coming decade (as a reasonable horizon). From a gastroenterologist's standpoint, diverticulitis is generally considered to be a minor disease, since the gastroenterologist is merely relegated to documenting the disease between episodes, with general surgeons currently entrusted with its definitive treatment. Selecting patients for what is, in essence, prophylactic surgery has been on the basis of quantitative discrimination (three strikes and it is out) or phenotypic behavior (unresolved inflammation, persistent symptoms, or complicated disease). Once the Cinderella disease of the gastrointestinal tract that has been all too often forgotten in research funding, some clarity has emerged from high-resolution qualitative analysis of patients with diverticulitis. Is diverticulitis a hereditary condition? That there are specific genetic signatures points to that assumption [1], representing a giant leap from the implied hereditary over environmental factors derived from epidemiologic data. Several decades ago, Levy and colleagues published two papers documenting that Ashkenazi Jews (16.2\%), Sephardic Jews (3.8\%), and Arabs $(0.7 \%)$ living in Israel had different prevalence of diverticulosis [2, 3]. It is tantalizing to think that a genetic signature could assist with risk stratification in order to inform surgical decision making. These findings were further expanded by the article by Kline et al. published in this issue of Digestive Diseases and Sciences [1] in which the authors identified key differences between patients with conventional and multifocal diverticulitis using RNA sequencing. In total, 69 genes had different expression in multifocal diverticulitis; in particular, those related to immunoresponsive behavior were at lower levels. This is a giant leap forward in the field with a tantalizing glimpse at the molecular processes that

Des C. Winter

des.winter@gmail.com

1 St Vincent's University Hospital, Elm Park, Dublin D04T6F4, Ireland determine who gets diverticulitis, why it may be multicentric, and how it may be a more aggressive phenotype in some. It is intuitive that there is an underlying genetic component, given the lack of robust evidence that environmental factors exert a direct causal influence, but these results are so startlingly clear in pointing to dysregulated immunity that it may be the key to unlocking the heterogeneity of diverticulitis. Is this the cusp of a new era where personalized care will include genetic profiling to predict the risk of recurrent or more aggressive diverticulitis? If so, it is a very welcome development that will be practice changing.

Of course, what is missing has been a medical option for patients wishing to avoid surgery. Perhaps the focus of drug trials has been incorrect in using anti-inflammatory, probiotic, or antibiotic medications. Should they have considered hypothetical agents that alter the genotypic-defined predilection for diverticulitis and how might that be achieved? Until there are some answers to those questions, medical therapy remains the tragic opera once used to describe surgical trials (full of promise and hope but ultimately disappointing). Minimally invasive surgical approaches made that more acceptable to patients with less morbidity and improved quality of life than what is, after all, termed "conservative management," basically a lifestyle intervention [4]. By better understanding the individual patient factors that may lead to complicated disease, prophylactic surgery may soon be considered at an earlier stage than is currently recommended, when the operation (and therefore anticipated recovery) should be more straightforward with less risk to surrounding structures. This may be the glass slipper patients and surgeons have been searching for.

\section{References}

1. Kline BP, Schiefer KM, Choi CS, et al. Multifocal vs conventional unifocal diverticulitis: a comparison of clinical and transcriptomic 
characteristics. Dig Dis Sci. (Epub ahead of print). https://doi. org/10.1007/s10620-018-5403-y.

2. Levy N, Luboshitzki R, Shiratzki Y, et al. Diverticulosis of the colon in Israel. Dis Colon Rectum. 1977;20:477-481.

3. Levy N, Stermer E, Simon J. The changing epidemiology of diverticular disease in Israel. Dis Colon Rectum. 1985;28:416-418.

4. Bolkenstein HE, Consten ECJ, van der Palen J, et al. Long-term outcome of surgery versus conservative management for recurrent and ongoing complaints after and episode of diverticulitis:
5 year follow-up results of a multicenter randomized controlled trial (DIRECT Trial). Ann Surg. 2019;269:612-620.

Publisher's Note Springer Nature remains neutral with regard to jurisdictional claims in published maps and institutional affiliations. 